\title{
Enzyme targets for drug design of new anti-virulence therapeutics
}

By

Charlene M. Kahler ${ }^{2}$ Mitali Sarkar-Tyson² Emily A. Kibble ${ }^{2}$ Keith A. Stubbs ${ }^{1}$ and Alice Vrielink $^{1,2}$

${ }^{1}$ School of Molecular Sciences, University of Western Australia, 35 Stirling Highway, Crawley, WA, 6009, Australia.

${ }^{2}$ Marshall Centre for Infectious Disease Research and Training, School of Biomedical Sciences, University of Western Australia, 35 Stirling Highway, Crawley, WA, 6009, Australia. 


\begin{abstract}
Society has benefitted greatly from the use of antibiotics. Unfortunately, the misuse of these valuable molecules has resulted in increased levels of antibiotic resistance, a major global and public health issue. This resistance and the reliance on a small number of biological targets for the development of antibiotics emphasizes the need for new targets. A critical aspect guiding the development of new antimicrobials through a rational structure-guided approach is to understand the molecular structures of specific biological targets of interest. Here we give an overview of the structures of bacterial virulence enzyme targets involved in protein folding, peptidoglycan biosynthesis and cell wall modification. These include enzymes of the thiol-disulphide oxidoreductase pathway (DSB enzymes), peptidyl-proly cis/trans isomerases (Mips), enzymes from the Mur pathway and enzymes involved in lipopolysaccharide modification (EptA and ArnT). We also present progress towards inhibitor design of these targets for the development of novel anti-virulence therapeutic agents.
\end{abstract}

\title{
Highlights
}

- Virulence enzymes are novel targets for development of antimicrobials

- Screening and rational design have identified inhibitors of DSB and Mip enzymes

- Transition state analogues are being developed to inhibit the Mur pathway

- EptA and ArnT inhibitors will demask bacteria for clearance by immune defensins 


\section{Introduction}

The discovery, development and utility of antibiotics to treat bacterial infections has arguably been one of the most successful public health activities of the $20^{\text {th }}$ century resulting in a dramatic increase in the quality of life for mankind. Antibiotics have been developed to treat a wide variety of Gram-positive and Gram-negative bacterial infections but their successful use has come at a cost, with an increasing number of reports of bacteria found to be resistant to current antibiotic therapies. Concurrently with this increase in antibiotic resistance there has been a decrease in the rate of development of new antimicrobials from the pharmaceutical industry $[1,2]$, resulting in a significant antibiotic crisis. Resistance has been reported for all current antibiotics including the $\beta$-lactams, glycopeptides, aminoglycosides, macrolides, quinolones, tetracyclines and alarmingly, even the last line antibiotics such as polymyxin [3]. As a result, the World Health Organisation and the Centres for Disease Control and Prevention in the USA have released a series of reports highlighting the global priority list of antimicrobial resistant pathogens and the urgency for the development of new treatment strategies including drug discovery to circumvent the problem with increasing antibiotic resistance $\left[4-7^{*}\right]$. One area of focus is to target bacterial virulence factors which are the cause of pathogenicity in the organism. Anti-virulence factors act to disarm the pathogen enabling clearance by the host immune system. Structures of new targets provide an important tool to assist in the development of novel antibacterial agents, allowing for screening and optimization using both fragment-based methods as well as established small molecule libraries. Two areas where development of new types of antimicrobials have been enhanced through the use of structural studies include enzymes involved in protein folding pathways as well as peptidoglycan biosynthesis and cell wall modification. Here we review the structures of a number of enzyme targets in these different pathways. Disulphide interchange and 
macrophage infectivity potentiator enzymes have already progressed with inhibitor design studies while the enzymes involved in peptidoglycan biosynthesis such as those of the Mur pathway and endotoxin modifying enzymes such as ArnT and EptA are more novel targets which are certain to be the extensive subject of further studies into inhibitor design.

\section{Disulphide Interchange Enzymes}

Protein synthesis and correct folding often requires the assistance of various chaperones including foldases (Hsp70 and Hsp100) [8], oxidoreductases and peptidyl proline cis-trans isomerases. Thiol-disulphide oxidoreductases (TDORs) are a large superfamily of enzymes that catalyze the oxidation, reduction, and isomerization of protein disulphide bonds [9]. All members of this family have an active site motif consisting of two Cys residues separated by two amino acids (CXXC) embedded in a thioredoxin-like fold [10]. TDORs are multi-functional and are widely dispersed in Gram-negative bacteria but have only recently been identified in Gram-positive bacteria through their involvement with specialised processes such as sporulation [11]. While cytoplasmic TDORs, such as thioredoxin, maintain a reducing environment inside the cell, extracytoplasmic TDORs in the cell envelope participate in a variety of physiological processes and in many bacterial pathogens they are essential for virulence [12].

The development of anti-virulence strategies by inhibiting TDORs has focused especially on the oxidative and isomerization periplasmic protein folding pathway in Gram-negative bacteria. The oxidation pathway consists of the disulphide bond (Dsb) proteins DsbA and DsbB, responsible for the catalysis of disulphide bond formation between adjacent Cys residues of protein substrates while the isomerization pathway (DsbC/DsbD system) reshuffles any incorrectly formed disulphide bonds (Figure 1a). DsbA is the primary disulphide 
donor that interacts with these substrates and is kept in an oxidized and active state by the membrane bound DsbB [13]. DsbA is a monomeric $21 \mathrm{kDa}$ periplasmic enzyme consisting of the thioredoxin fold containing an extra alpha-domain (65 residues forming four $\alpha$-helices) inserted into the center of the thioredoxin domain (Figure 1b). The $\alpha$-domain forms a globular-like cap over the active site CXXC motif located at the $\mathrm{N}$-terminus of the first $\alpha$-helix of the thioredoxin domain [14]. The redox activity of the enzymes is highly modulated by the presence of a cis proline containing loop that makes van der Waals interactions with the active site cysteine residues [15]. A hydrophobic groove, critical for binding to DsbB during the catalytic cycle, has been targeted as a potential inhibitor binding site $[16,17]$. DsbB is a cytoplasmic membrane protein and a member of the vitamin $\mathrm{K} 2,3$-epoxide reductase (VKOR) superfamily; it contains four trans-membrane $\alpha$-helices connected by two periplasmic loops (P1 and P2) that house two cysteine pairs (Cys41-Cys44 and Cys104-Cys130 respectively) essential to DsbA oxidation [18,19] (Figure 1c). The NMR structure of DsbB [20] as well as the crystal structure of the complex of DsbA and DsbB gives important insights into the interaction of the two proteins and the mechanism of rapid DsbB-mediated oxidation of DsbA $\left[21^{*}, 22^{* *}\right]$. The transfer of electrons from DsbA to DsbB is initiated by interaction of the P2 loop of DsbB with the open hydrophobic groove of reduced DsbA (Figure 1c). This induces a conformational change in the P2 loop of DsbB so that Cys104-Cys130 are separated spatially, and brought into contact with the reduced cysteines (Cys30 and Cys44) of the DsbA active site, enabling a rearrangement of the disulphide bonds and the subsequence electron transfer to the ubiquinone moiety bound to DsbB through the formation of a Cys44-ubiquinone charge transfer complex.

DsbC is a V-shaped homodimer consisting of two $23 \mathrm{kDa}$ monomers (Figure 1d). The monomers consists of a C-terminal thioredoxin domain and an $\mathrm{N}$-terminal dimerization 
domain connected by a linker sequence [23*]. The active site residues in the thioredoxin domain are positioned so as to face the inside hydrophobic surface of the V-shaped homodimeric structure and prevents interaction with DsbB [23*]. Oxidized DsbC is activated by reduction from the inner membrane bound $\mathrm{DsbD}$ which mediates electron transfer from cytoplasmic thioredoxin [24]. DsbD consists of a periplasmic N-terminal domain with an immunoglobulin-like fold (DsbDa), a hydrophobic core that contains eight transmembrane helices (DsbD $\beta$ ) and periplasmic C-terminal domain (DsbDy) with a thioredoxin-like fold. The sequence of electron transfer through these domains is $\mathrm{DsbD} \beta \rightarrow \mathrm{DsbD} \gamma \rightarrow \mathrm{DsbD} \alpha$ [25] (Figure 1a). The electrons are then further transferred from $\mathrm{DsbD} \alpha$ to DsbC. The structure of the DsbC-DsbDa domain shows two asymmetric binding sites with the primary binding site forming an intermolecular disulphide bond between Cys98 of DsbC and Cys109 of $\operatorname{DsbD} \alpha\left[26^{* *}\right]$ (Figure $\left.1 d^{* *}\right)$. Furthermore, the DsbC binding cleft undergoes conformational changes upon DsbDa binding to a closed conformation which has led to a model for the interaction of $\mathrm{DsbC}$ to other protein substrates for isomerisation activity.

Although the Dsb pathway has not been found to be essential in Gram-negative bacteria [9, 27], inactivation of the pathway has been shown to attenuate all Gram-negative pathogens investigated to date $[2,28]$. The structures of the different components of the Dsb pathway open up possibilities for structure-guided design of anti-virulence agents that will assist the innate immune system in clearing the bacterial infection. Anti-virulence compound design has focused mainly on the oxidation pathway (DsbA, DsbB). Rational target-based drug design (RTBDD) strategies [28] have successfully identified novel small molecules that inhibit the function of DsbA and DsbB. Using a combination of STD-NMR based fragment screening followed up by chemical elaboration, small molecules have been identified [29**] that bind to the active site hydrophobic groove, inhibiting DsbA activity in Escherichia coli by blocking 
the DsbB peptide binding site [16*] (Figure 1e). These studies provide a starting point for further inhibitor development as anti-virulence agents. Due to the structural groups of DsbA [30], opportunities may exist to create specialised inhibitors that target Enterobacteriaceae, an important group of pathogens most related to the spread of antimicrobial resistance.

\section{Peptidyl-proly cis/trans isomerase enzymes}

A novel group of enzymes involved in protein folding and essential for bacterial survival are the macrophage infectivity potentiator (Mip) proteins. Part of the immunophilin superfamily, Mip is a FK506 binding protein (FKBP) that exhibits peptidyl-prolyl cis/trans isomerase (PPlase) activity which catalyses the cis-trans isomerisation of peptidyl-proline residues, a rate-limiting reaction in the process of bacterial protein folding. As such, Mip proteins play a pleiotropic role in the virulence of several human pathogenic bacteria (reviewed in [31*]). Although the exact targets for Mips in bacteria are yet to be elucidated, novel inhibitors have been generated with potent binding to recombinant Mips from a number of different bacterial species.

While all Mips share a similar C-terminal, FKBP catalytic domain, Legionella pneumophila Mip (LpMip) exists as a homodimer whereas Burkholderia pseudomallei Mip (BpML1) and Trypanosoma cruzi Mip (TcMip) are monomers. Homodimerization is mediated by an Nterminal domain which is connected via a long alpha helix to a $C$ terminal FKBP like domain which contains the rotamase active site (Figure 2a). This domain is composed of six $\beta$-strands forming an antiparallel sheet with a short $\alpha$-helix lying across the concave surface of the sheet. The active site is a predominantly hydrophobic surface located between this helix and the concave surface of the $\beta$-sheet (Figure $2 b$ ) and is highly conserved across the entire FKBP family. Residues making up the substrate binding site are localized in the helix and strands of 
the sheet as well as the $\beta 4-\beta 5$ loop. The conformations of side chains in the substrate binding pocket are found to be more similar in BpML1 and TcMip while the active site of BpML1 is more flexible with the active site Tyr89 in the $\beta 4-\beta 5$ loop adopting two different conformations. The similarity and activity of these proteins has been confirmed through infection models, with LpMip functionally complementing TcMip when expressed in a T. cruzi Mip mutant strain [32]. These studies suggest that Mip proteins have considerable potential as broad-spectrum anti-virulence targets especially due to the conserved catalytic FKBP domain as demonstrated by the activity of Mip inhibitors in a range of Gram-negative bacteria.

Mip inhibitors have been designed, based on the macrolide immunosuppressive drugs FK506 and rapamycin, which are potent inhibitors of the PPlase activity of Mip proteins [33]. FK506 and rapamycin both possess a key pipecolinyl ring system that mimics a peptidyl-prolyl bond and bind to a hydrophobic pocket of the active site of Mip (Figure 2c). The remaining part of the inhibitors faces away from the protein and is involved in the immune-modulatory side effects of these drugs [34]. The two discrete portions of these inhibitors allowed for the design of drugs based on the core inhibitory portion and not the portion responsible for the immune-modulatory effect. This has led to the generation of small molecule inhibitors, based on the pipecolic ring structure of rapamycin, against the LpMip and BpML1 $\left[35^{*}, 36\right]$ (Figure 2d) Although these inhibitors reduced enzymatic activity of LpMip, there was no effect on $L$. pneumophila replication in macrophages [36]. This was not the case with BpML1 where enzymatic activity was inhibited and a reduction in bacterial-induced cytotoxicity in macrophages was observed $[35,37]$. Furthermore, using in vitro cell infection models, similar inhibitors have been shown to have negative effects on the developmental cycle of Chlamydia trachomatis, reduced survival of Neisseria gonorrohoeae in neutrophils, and reduction in 
adherence and invasion of Neisseria meningitidis in epithelial cells [38]. These results provide compelling evidence of the broad-spectrum activity of Mip inhibitors against a range of Gramnegative bacteria. As an anti-virulence target, Mip inhibition may result in debilitating the bacteria, allowing the host immune system to clear infection more efficiently, or alternatively could be used in combination with antibiotics.

\section{Peptidoglycan Biosynthesis Targets}

The peptidoglycan is an important component of the bacterial cell wall and an essential structure that provides protection from the external environment as well as strength and rigidity to combat internal osmotic pressure. Both Gram-positive and Gram-negative bacteria have structurally similar peptidoglycan however they differ in the extent of cross-linking and thickness. Due to the lack of this structure in eukaryotes, the enzymes involved in the biosynthesis and in the modification of this cell wall component have acted as targets for antimicrobial drug development. The most notable example are enzymes involved in peptidoglycan cross linking such as the penicillin-binding proteins, the target of $\beta$-lactams antibiotics (see review by Bush and Bradford [39]). There is continued interest in peptidoglycan biosynthesis which involves a number of different steps using six enzymes, MurA-F, to ultimately form the peptidoglycan building block, UDP-Mur-Nacetylmuramoyl:pentapeptide. These early steps of peptidoglycan biosynthesis have, as yet, not been exploited in drug development in any great detail. Fosfomycin has been developed to inhibit MurA responsible for the ligation of phosphoenolpyruvate to UDP-Nacetylglucosamine [40]. More recently, structures of MurC from $\mathrm{H}$. influenzae and $E$. coli have been reported $[41,42]$ enabling a structure-based drug design approach against this target. 
MurC carries out the ATP dependent ligation of L-alanine and UDP-N-acetylmuramic acid to form UDP-N-acetylmuramoyl-L-alanine with allows for further ligations catalysed by MurD, MurE and MurF (Figure 3). All four Mur ligases (MurC-F) share common features: a divalent cation such as $\mathrm{Mg}^{2+}$ or $\mathrm{Mn}^{2+}, 6$ invariant residues and an ATP-binding consensus sequence. These enzymes also adopt highly conserved three-dimensional structures: an N-terminal domain involved in UDP substrate binding, a central ATPase domain and a C-terminal domain, associated with binding of the incoming amino acid (Figure 3b). The structures of MurC are reported in the apo form [41] with a single $\mathrm{Mg}^{2+}$ bound as well as in complex with a nonhydrolysable ATP analogue, AMPPNP, and UDP-N-acetylmuramic acid (UNAM) in which case two $\mathrm{Mn}^{2+}$ ions are present $\left[42^{*}\right]$. The bound ligands are found in an extensive cavity: the AMPPNP binding site is positioned between the central ATPase domain and the C-terminal domain while the UNAM binding site lies along the inner surface of the N-terminal UDP substrate binding domain (Figure 3c). This cavity presents an opportunity for inhibitor design which exploits both binding sites simultaneously. Phosphinate inhibitors to MurC are the most potent examples of amino acid ligase inhibitors known to date. These act as putative transition state mimics of the enzyme however use of these inhibitors failed to show antibacterial activity presumably due to the inability of the molecules to be transported to their site of action. A high throughput screen of compounds based at AstraZeneca identified the pyrazolopyrimidine scaffold as a MurC inhibitor class [43] and has been suggested as a starting point for further optimization however the mode of binding of the compound to the enzyme has, to date, not been established. Given the similarity of the structures and chemistry of the MurC-F enzymes, inhibitors designed to target one of the enzymes may also inhibit, even moderately, the other ligases thus providing an additional level of inhibition of the pathway. Putative transition state analogues targeting MurD consisting of naphthalene- 
$\mathrm{N}$-sulfonyl-D-Glu motifs have been shown to exhibit $\mathrm{IC}_{50}$ values in the micromolar range. Structures of MurD-inhibitor complexes show they bind within the cavity, predominantly at the UDP-binding domain of the enzyme (Figure 3d) and suggest excellent starting points for further inhibitor optimization $\left[44^{*}, 45\right]$ with the goal of modifying the scaffold to target the other Mur enzymes.

\section{Cell Wall Modification Enzymes}

The cell membrane of many pathogenic Gram-negative bacteria is comprised in part of lipopolysaccharides (LPS). These complex molecules form a barrier allowing the bacteria to survive in harsh environments $[46,47]$ and protect the organism from antibiotics [48-50] and immune system defensins [51]. As such, LPS is essential for the survival of many Gramnegative bacteria $[52,53]$ and studies have shown that LPS-defective mutants have increased susceptibility to antibiotics. Enzymes that modify the LPS, and particularly the lipid A moiety are considered excellent targets for novel antimicrobial therapeutic development. Modifications include the addition of phosphoethanolamine moieties to Lipid A by the enzyme EptA [54-56] and the addition of 4-amino-4-deoxy-L-arabinose (L-Ara4N) to lipid A phosphate groups by the enzyme ArnT [57] (Figure 4a). These modifications reduce the negative charge on the outer membrane of the bacteria thus decreasing the interaction of positively charged antibiotics such as colistin and polymyxin as well as host innate immune defensins [58]. Recently, Mcr-1 (mobile colistin resistance), an EptA homologue, has been found on a mobilizable plasmid [59**-61]. ArnT, EptA and Mcr-1 are integral membrane proteins, found in the inner membrane of Gram-negative bacteria. Recent structures of the periplasmic domains of EptA [62] and Mcr-1 [63-66], full-length ArnT [67**] (Figure 4b) and full-length EptA [68**] (Figure 4d) provide a basis for future structure-guided drug design. 
The substrate binding sites of the enzymes are positioned at the interface between the membrane and periplasmic domains and contain a $\mathrm{Zn}^{2+}$ ion coordinated by invariant residues. In the case of ArnT, the presence of a bound undecaprenyl phosphate (UndP) results in a rearrangement of a periplasmic loop (PL4) and the loss of the $\mathrm{Zn}^{2+}$ ion (Figure 4c). This supports the proposed role of the metal ion in orienting the PL4 loop conformation so as to allow UndP substrate binding. The position of the bound UndP and the proposed position of Lipid A in a large cavity accessible to the outer leaflet of the inner membrane with the conserved active site residues located between these binding sites (Figure 4c) has led to the hypothesis that the substrates bind sequentially with the donor substrate, undecaprenyl phosphate- $\alpha$-4-amino-4-deoxy-L-arabinose (UndP- $\alpha$-L-Ara4N), stabilizing a conformation that enables Lipid A to bind. Transfer of 4-amino-4-deoxy-L-arabinose (L-Ara4N) putatively occurs directly from UndP to Lipid A without the formation of an intermediate. This concerted mechanism is consistent with the glycosidic bond inversion reported for L-Ara4N transfer to Lipid A [69].

In contrast, the recently determined structure of full-length EptA [68] (Figure 4d) only reveals a binding site that facilitates the binding of the donor substrate, phosphatidylethanolamine. A cavity large enough to bind Lipid A is notably absent in the structure. However, EptA is proposed to go through a ping-pong catalytic mechanism involving the formation of an enzyme PEA intermediate using a catalytic threonine residue (Figure 4e). The structure of the enzyme and molecular dynamic studies suggest a large conformational change reorienting the periplasmic relative to the membrane domain, resulting in a more open structure that may facilitate binding of the Lipid A acceptor substrate for PEA transfer from the PEA bound enzyme intermediate. 
In both ArnT and EptA, the structures and a detailed understanding of the mechanism of substrate binding and catalysis will enable a structure guided approach to inhibitor design. In the case of EptA, fragment screening studies and chemical elaboration has already identified inhibitors of the enzyme leading to increased polymyxin sensitivity of Neisseria gonorrhoeae. The molecular mechanism of inhibition by these compounds through structural studies is a current focus of work in our group. Inhibition of both ArnT and EptA will constitute an important advance in reversing polymyxin resistance as well as demasking Gram-negative bacteria to enable clearance by the host immune system.

\section{Conclusions}

The global increase in antibiotic resistance highlights the urgent need for the development of novel antimicrobial therapies. Virulence causing enzymes in bacteria are seen as important targets for the development of novel antimicrobial agents. These targets include protein folding enzymes responsible for correct disulphide bond formation and peptidyl proline bond conformation. Progress towards inhibitor design of these enzyme targets has progressed starting with fragment-based screening methods as well as rational structure guided drug design strategies. Additionally, peptide-based design to block DsbA/DsbB interaction is an active area of inhibitor design. The Mur pathway of enzymes involved in the early stages of peptidoglycan biosynthesis are being targeted for inhibitor development through the design of transition state analogues. The structural similarity of the MurC-F enzymes suggests that inhibitors of one enzyme may be modified slightly to target other enzymes in the pathway. Finally, two new membrane bound enzymes involved in lipopolysaccharide modification, EptA and ArnT, are important targets for inhibitor design to alter the charged state of the 
bacterial wall thus enabling clearance by host innate immune defensins as well as last line antibiotics such as colistin and polymyxin.

\section{Acknowledgements}

This work was supported by National Health and Medical Research Council of Australia Grants (APP1003697 (to A.V. and C.M.K.) and APP1078642 (to A.V., C.M.K., and K.A.S.).

\section{References and recommended reading}

Papers of particular interest, published within the period of review have been highlighted as:

* of special interest

** of outstanding interest

1. Projan SJ: Why is big Pharma getting out of antibacterial drug discovery? Curr Opin Microbiol 2003, 6:427-430.

2. Power E: Impact of antibiotic restrictions: the pharmaceutical perspective. Clin Microbiol Infect 2006, 12 Suppl 5:25-34.

3. Fair RJ, Tor Y: Antibiotics and bacterial resistance in the 21st century. Perspect Medicin Chem 2014, 6:25-64.

4. Centers for Disease Control and Prevention: Sexually transmitted diseases surveillance 2010. Edited by; 2011.

5. Centers for Disease Control and Prevention: Antibiotic resistance threats in the United States, 2013. Edited by: US Department of Health and Human Services; 2013:1-114. 
6. World Health Organization: Antimicrobial resistance: global report on surveillance.

Edited by. Geneva: World Health Organisation; 2014:1-256.

7. World Health Organization: Global priority list of antibiotic-resistant bacteria to guide research, discovery, and development of new antibiotics. Edited by. Geneva: World Health Organization; 2017:1-7.

* Discussion paper released by the World Health Organisation highlighting the threats that bacteria have on public health as well as discussion of pathogens that present a serious threat to public health.

8. Mogk A, Bukau B, Kampinga $\mathrm{HH}$ : Cellular handling of protein aggregates by disaggregation machines. Mol Cell 2018, 69:214-226.

9. Landeta C, Boyd D, Beckwith J: Disulfide bond formation in prokaryotes. Nat Microbiol 2018, 3:270-280.

10. Martin JL: Thioredoxin--a fold for all reasons. Structure 1995, 3:245-250.

11. Davey L, Halperin SA, Lee SF: Thiol-disulfide exchange in Gram-positive firmicutes. Trends Microbiol 2016, 24:902-915.

12. Heras B, Shouldice SR, Totsika M, Scanlon MJ, Schembri MA, Martin JL: DSB proteins and bacterial pathogenicity. Nat Rev Microbiol 2009, 7:215-225.

13. Bardwell JC, Lee JO, Jander G, Martin N, Belin D, Beckwith J: A pathway for disulfide bond formation in vivo. Proc Natl Acad Sci U S A 1993, 90:1038-1042.

14. Martin JL, Bardwell JC, Kuriyan J: Crystal structure of the DsbA protein required for disulphide bond formation in vivo. Nature 1993, 365:464-468.

15. Charbonnier JB, Belin P, Moutiez M, Stura EA, Quemeneur E: On the role of the cisproline residue in the active site of DsbA. Protein Sci 1999, 8:96-105. 
16. Duprez W, Premkumar L, Halili MA, Lindahl F, Reid RC, Fairlie DP, Martin JL: Peptide inhibitors of the Escherichia coli DsbA oxidative machinery essential for bacterial virulence. J Med Chem 2015, 58:577-587.

* Structure activity studies of DsbB derived peptide to probe their interaction with DsbA. This work includes biophysical studies to assess a panel of peptides and a structure of one of the peptides complexed to DsbA. The study provides evidence that Peptides could be used to interfere with the DsbA-DsbB binding site.

17. McMahon RM, Ireland PM, Sarovich DS, Petit G, Jenkins CH, Sarkar-Tyson M, Currie BJ, Martin JL: Virulence of the melioidosis pathogen Burkholderia pseudomallei requires the oxidoreductase membrane protein DsbB. Infect Immun 2018, 86.

18. Jander G, Martin NL, Beckwith J: Two cysteines in each periplasmic domain of the membrane protein DsbB are required for its function in protein disulfide bond formation. EMBO J 1994, 13:5121-5127.

19. Kadokura H, Beckwith J: Four cysteines of the membrane protein DsbB act in concert to oxidize its substrate DsbA. EMBO J 2002, 21:2354-2363.

20. Zhou Y, Cierpicki T, Jimenez RH, Lukasik SM, Ellena JF, Cafiso DS, Kadokura H, Beckwith J, Bushweller JH: NMR solution structure of the integral membrane enzyme DsbB: functional insights into DsbB-catalyzed disulfide bond formation. Mol Cell 2008, 31:896-908.

21. Inaba K, Murakami S, Nakagawa A, lida H, Kinjo M, Ito K, Suzuki M: Dynamic nature of disulphide bond formation catalysts revealed by crystal structures of DsbB. EMBO J 2009, 28:779-791. 
* Presents the structure of a DsbB-Fab complex and gives an updated overview of the DsbBDsbA complex. The results suggest that DsbB has the ability to change its conformation dynamically in response to DsbA binding.

22. Inaba K, Murakami S, Suzuki M, Nakagawa A, Yamashita E, Okada K, Ito K: Crystal structure of the DsbB-DsbA complex reveals a mechanism of disulfide bond generation. Cell 2006, 127:789-801.

** The first crystal structure of the DsbA-DsbB complex giving structural insights into the mechanism of intermolecular disulfide interchange between the two oxidoreductase partners.

23. McCarthy AA, Haebel PW, Torronen A, Rybin V, Baker EN, Metcalf P: Crystal structure of the protein disulfide bond isomerase, DsbC, from Escherichia coli. Nat Struct Biol 2000, 7:196-199.

*Molecualr view of DsbC showing the hinged domain arrangement and the V-shaped surface cleft for providing insights for protein binding and refolding through disulfide bond formation.

24. Rietsch A, Bessette P, Georgiou G, Beckwith J: Reduction of the periplasmic disulfide bond isomerase, DsbC, occurs by passage of electrons from cytoplasmic thioredoxin. J Bacteriol 1997, 179:6602-6608.

25. Katzen F, Beckwith J: Transmembrane electron transfer by the membrane protein DsbD occurs via a disulfide bond cascade. Cell 2000, 103:769-779.

26. Haebel PW, Goldstone D, Katzen F, Beckwith J, Metcalf P: The disulfide bond isomerase DsbC is activated by an immunoglobulin-fold thiol oxidoreductase: crystal structure of the DsbC-DsbDalpha complex. EMBO J 2002, 21:4774-4784. 
** Crystal structure of the complex between DsbC and the alpha domain of DsbD. This structure shows that $\mathrm{DsbD} \alpha$ is a novel oxidoreductase with the active site cysteine residues positioned in an immunoglobulin fold. Also the structure shows the DsbDa located in the central cleft of the DsbC dimer which adopts a closed conformation relative to the uncomplexed state. This gives insights into the role DsbD plays in selectively activating DsbC. 27. Kumar P, Sannigrahi S, Scoullar J, Kahler CM, Tzeng YL: Characterization of DsbD in Neisseria meningitidis. Mol Microbiol 2011, 79:1557-1573.

28. Smith RP, Paxman JJ, Scanlon MJ, Heras B: Targeting bacterial Dsb proteins for the development of anti-virulence agents. Molecules 2016, 21.

29. Adams LA, Sharma P, Mohanty B, Ilyichova OV, Mulcair MD, Williams ML, Gleeson EC, Totsika M, Doak BC, Caria S, et al.: Application of fragment-based screening to the design of inhibitors of Escherichia coli DsbA. Angew Chem Int Ed Engl 2015, $54: 2179-2184$.

** Discusses the development of potent inhibitors of DsbA using a NMR-based fragment screening method. Fragments were assessed using biophysical methods and one compound elaborated to give compounds which inhibit motility but do not effect growth, consistent with on-target effects against DsbA.

30. Totsika M, Vagenas D, Paxman JJ, Wang G, Dhouib R, Sharma P, Martin JL, Scanlon MJ, Heras B: Inhibition of diverse DsbA enzymes in multi-DsbA encoding pathogens. Antioxid Redox Signal 2018, 29:653-666.

31. Unal CM, Steinert M: Microbial peptidyl-prolyl cis/trans isomerases (PPlases): virulence factors and potential alternative drug targets. Microbiol Mol Biol Rev 2014, 78:544-571. 
* A comprehensive review of the superfamily of peptidyl-prolys cis/trans isomerases and their wide role in biological processes. Discussion of their classification as virulence associated proteins is also discussed.

32. Pereira PJB, Vega MC, González-Rey E, Fernández-Carazo R, Macedo-Ribeiro S, GomisRüth FX, González A, Coll M: Trypansoma cruzi macrophage infectivity potentiator has a rotamase core and a highly exposed a-helix. EMBO Reports 2002, 3:88-94.

33. Hacker J, Fischer G: Immunophilins: structure-function relationship and possible role in microbial pathogenicity. Mol Microbiol 1993, 10:445-456.

34. Van Duyne GD, Standaert RF, Karplus PA, Schreiber SL, Clardy J: Atomic structure of FKBP-FK506, an immunophilin-immunosuppressant complex. Science 1991, 252:839-842.

35. Begley DW, Fox D, 3rd, Jenner D, Juli C, Pierce PG, Abendroth J, Muruthi M, Safford K, Anderson V, Atkins K, et al.: A structural biology approach enables the development of antimicrobials targeting bacterial immunophilins. Antimicrob Agents Chemother 2014, 58:1458-1467.

* Discusses the development of potent inhibitors of Mips. Using a combination of X-ray crystallographic and and NMR studies a key scaffold was identified. This scaffold was elaborated to generate compounds that exhibited in vitro activity against $B$. pseudomallei. 36. Juli C, Sippel M, Jager J, Thiele A, Weiwad M, Schweimer K, Rosch P, Steinert M, Sotriffer CA, Holzgrabe U: Pipecolic acid derivatives as small-molecule inhibitors of the Legionella MIP protein. J Med Chem 2011, 54:277-283.

37. Seufert F, Kuhn M, Hein M, Weiwad M, Vivoli M, Norville IH, Sarkar-Tyson M, Marshall LE, Schweimer K, Bruhn H, et al.: Development, synthesis and structure-activityrelationships of inhibitors of the macrophage infectivity potentiator (Mip) proteins 
of Legionella pneumophila and Burkholderia pseudomallei. Bioorg Med Chem 2016, 24:5134-5147.

38. Reimer A, Seufert F, Weiwad M, Ebert J, Bzdyl NM, Kahler CM, Sarkar-Tyson M, Holzgrabe U, Rudel T, Kozjak-Pavlovic V: Inhibitors of macrophage infectivity potentiator-like PPlases affect neisserial and chlamydial pathogenicity. Int J Antimicrob Agents 2016, 48:401-408.

39. Bush K, Bradford PA: Beta-lactams and beta-lactamase Inhibitors: An overview. Cold Spring Harb Perspect Med 2016, 6.

40. Hendlin D, Stapley EO, Jackson M, Wallick H, Miller AK, Wolf FJ, Miller TW, Chaiet L, Kahan FM, Foltz EL, et al.: Phosphonomycin, a new antibiotic produced by strains of Streptomyces. Science 1969, 166:122-123.

41. Deva T, Baker EN, Squire CJ, Smith CA: Structure of Escherichia coli UDP-Nacetylmuramoyl:L-alanine ligase (MurC). Acta Crystallogr D Biol Crystallogr 2006, 62:1466-1474.

42. Mol CD, Brooun A, Dougan DR, Hilgers MT, Tari LW, Wijnands RA, Knuth MW, McRee DE, Swanson RV: Crystal structures of active fully assembled substrate- and productbound complexes of UDP-N-acetylmuramic acid:L-alanine ligase (MurC) from Haemophilus influenzae. J Bacteriol 2003, 185:4152-4162.

* Crystal structures of MurC from Haemophilus influenzae in complex with the substrate UDP-N-aceylmuramic acid and $\mathrm{Mg}^{2+}$ as well as a ternary complex with the product, UDP-Nacetylmuramoyl-L-alanine and the non hydrolyzable ATP analogue, AMPPNP. Structural comparisons with MurD suggest possibilities to target specific enzymes in the Mur pathway. 43. Humnabadkar V, Prabhakar KR, Narayan A, Sharma S, Guptha S, Manjrekar P, Chinnapattu M, Ramachandran V, Hameed SP, Ravishankar S, et al.: UDP-N- 
acetyImuramic acid I-alanine ligase (MurC) inhibition in a tolC mutant Escherichia coli strain leads to cell death. Antimicrob Agents Chemother 2014, 58:6165-6171.

44. Humljan J, Kotnik M, Contreras-Martel C, Blanot D, Urleb U, Dessen A, Solmajer T, Gobec S: Novel naphthalene-N-sulfonyl-D-glutamic acid derivatives as inhibitors of MurD, a key peptidoglycan biosynthesis enzyme. J Med Chem 2008, 51:7486-7494.

* Reports on the development of transition state analogues as inhibitors of MurD utilising a naphthalene core. Structural insight into reasons behind potency is described through the aid of co-crystal structures.

45. Kotnik M, Humljan J, Contreras-Martel C, Oblak M, Kristan K, Herve M, Blanot D, Urleb U, Gobec S, Dessen A, et al.: Structural and functional characterization of enantiomeric glutamic acid derivatives as potential transition state analogue inhibitors of MurD ligase. J Mol Biol 2007, 370:107-115.

46. Leive L: The barrier function of the Gram-negative envelope. Ann N Y Acad Sci 1974, 235:109-129.

47. Nikaido H: Molecular basis of bacterial outer membrane permeability revisited. Microbiol Mol Biol Rev 2003, 67:593-656.

48. Sanderson KE, MacAlister T, Costerton JW, Cheng KJ: Permeability of lipopolysaccharide-deficient (rough) mutants of Salmonella typhimurium to antibiotics, lysozyme, and other agents. Can J Microbiol 1974, 20:1135-1145.

49. Tamaki S, Sato T, Matsuhashi M: Role of lipopolysaccharides in antibiotic resistance and bacteriophage adsorption of Escherichia coli K-12. J Bacteriol 1971, 105:968975.

50. Vaara M: Antibiotic-supersusceptible mutants of Escherichia coli and Salmonella typhimurium. Antimicrob Agents Chemother 1993, 37:2255-2260. 
51. Lewis LA, Shafer WM, Dutta Ray T, Ram S, Rice PA: Phosphoethanolamine residues on the lipid A moiety of Neisseria gonorrhoeae lipooligosaccharide modulate binding of complement inhibitors and resistance to complement killing. Infect Immun 2013, 81:33-42.

52. Galloway SM, Raetz CR: A mutant of Escherichia coli defective in the first step of endotoxin biosynthesis. J Biol Chem 1990, 265:6394-6402.

53. Zhang G, Meredith TC, Kahne D: On the essentiality of lipopolysaccharide to Gramnegative bacteria. Curr Opin Microbiol 2013, 16:779-785.

54. Cox AD, Wright JC, Li J, Hood DW, Moxon ER, Richards JC: Phosphorylation of the lipid A region of meningococcal lipopolysaccharide: identification of a family of transferases that add phosphoethanolamine to lipopolysaccharide. J Bacteriol $2003,185: 3270-3277$.

55. Bartley SN, Kahler CM: The glycome of Neisseria spp.: How does this relate to pathogenesis? In Pathogenic Neisseria: Genomics, molecular biology, and disease prevention. Edited by Kahler CM, Davies JK: Caister Academic Press; 2014.

56. Needham BD, Trent MS: Fortifying the barrier: the impact of lipid A remodelling on bacterial pathogenesis. Nat Rev Microbiol 2013, 11:467-481.

57. Trent MS, Ribeiro AA, Lin S, Cotter RJ, Raetz CR: An inner membrane enzyme in Salmonella and Escherichia coli that transfers 4-amino-4-deoxy-L-arabinose to lipid A: induction on polymyxin-resistant mutants and role of a novel lipid-linked donor. J Biol Chem 2001, 276:43122-43131.

58. Pristovsek P, Kidric J: Solution structure of polymyxins B and E and effect of binding to lipopolysaccharide: an NMR and molecular modeling study. J Med Chem 1999, 42:4604-4613. 
59. Liu YY, Wang Y, Walsh TR, Yi LX, Zhang R, Spencer J, Doi Y, Tian G, Dong B, Huang X, et al.: Emergence of plasmid-mediated colistin resistance mechanism MCR-1 in animals and human beings in China: a microbiological and molecular biological study. Lancet Infect Dis 2016, 16:161-168.

** Demonstrates the emergence and mobility of Mcr-1 on a plasmid, the first plasmidmediated polymyxin resistance determinant. Using sequence homology and mass spectrometry the prevalence of the gene was investigated in various strains from various regions in China. This was the first study to demonstrate the mobility of this important resistance determinant.

60. McGann P, Snesrud E, Maybank R, Corey B, Ong AC, Clifford R, Hinkle M, Whitman T, Lesho E, Schaecher KE: Escherichia coli harboring mcr-1 and blaCTX-M on a novel IncF plasmid: First report of mcr-1 in the United States. Antimicrob Agents Chemother 2016, 60:4420-4421.

61. Rapoport M, Faccone D, Pasteran F, Ceriana P, Albornoz E, Petroni A, Group MCR, Corso A: First description of mcr-1-mediated colistin resistance in human infections caused by Escherichia coli in Latin America. Antimicrob Agents Chemother 2016, 60:4412-4413.

62. Wanty C, Anandan A, Piek S, Walshe J, Ganguly J, Carlson RW, Stubbs KA, Kahler CM, Vrielink A: The structure of the neisserial lipooligosaccharide phosphoethanolamine transferase $A$ (LptA) required for resistance to polymyxin. J Mol Biol 2013, 425:3389-3402.

63. Hinchliffe P, Yang QE, Portal E, Young T, Li H, Tooke CL, Carvalho MJ, Paterson NG, Brem J, Niumsup PR, et al.: Insights into the mechanistic basis of plasmid-mediated 
colistin resistance from crystal structures of the catalytic domain of MCR-1. Sci Rep $2017,7: 39392$.

64. Hu M, Guo J, Cheng Q, Yang Z, Chan EW, Chen S, Hao Q: Crystal Ssructure of Escherichia coli originated MCR-1, a phosphoethanolamine transferase for colistin resistance. Sci Rep 2016, 6:38793.

65. Ma G, Zhu Y, Yu Z, Ahmad A, Zhang H: High resolution crystal structure of the catalytic domain of MCR-1. Sci Rep 2016, 6:39540.

66. Stojanoski V, Sankaran B, Prasad BV, Poirel L, Nordmann P, Palzkill T: Structure of the catalytic domain of the colistin resistance enzyme MCR-1. BMC Biol 2016, 14:81.

67. Petrou VI, Herrera CM, Schultz KM, Clarke OB, Vendome J, Tomasek D, Banerjee S, Rajashankar KR, Belcher Dufrisne M, Kloss B, et al.: Structures of aminoarabinose transferase ArnT suggest a molecular basis for lipid A glycosylation. Science 2016, 351:608-612.

** Reports on the crystal structure of ArnT giving insights into the addition of 4-amino-4deoxy-L-arabinose to Lipid A. The structure with the lipid carrier (UndP) especially gives a rationale for transfer of the carbohydrate. A rationale for the catalytic mechaim is also described.

68. Anandan A, Evans GL, Condic-Jurkic K, O'Mara ML, John CM, Phillips NJ, Jarvis GA, Wills SS, Stubbs KA, Moraes I, et al.: Structure of a lipid A phosphoethanolamine transferase suggests how conformational changes govern substrate binding. Proc Natl Acad Sci U S A 2017, 114:2218-2223.

** Discusses the crystal structure of the full length EptA a phosphoethanolamine transferases that adds phosphoethanolamine to Lipid A. A molecular dynamics study of the 
enzyme also suggests conformational changes that provide a mechanistic basis for the ping pong catalytic mechanism.

69. Zhou Z, Ribeiro AA, Raetz CR: High-resolution NMR spectroscopy of lipid A molecules containing 4-amino-4-deoxy-L-arabinose and phosphoethanolamine substituents. Different attachment sites on lipid A molecules from NH4VO3-treated Escherichia coli versus kdsA mutants of Salmonella typhimurium. J Biol Chem 2000, 275:1354213551. 


\section{Figure Captions}

Figure 1. Thiol-disulphide oxidoreductase enzymes in bacteria. (a) Thiol-disulphide oxidoreductase protein folding pathway in the periplasm of Gram-negative bacteria. (b) Secondary structure representation of DsbA (PDB: 1DSB). The thioredoxin like domain is shown in red and the alpha domain is shown in green. The active site disulphide and the conserved cys-proline are shown in stick representation. (c) Structure of the DsbA-DsbB complex. DsbA is shown as a green surface. DsbB is shown in wheat coloured ribbon. The P1 loop on DsbB is coloured orange and the P2 loop is coloured pink. The bound ubiquinone (Ubq) is shown in ball and stick representation (PDB:2ZUP). (d). Ribbon representation of the DsbC-DsbD $\alpha$ complex. The catalytic domains of the DsbC monomers are shown in different shades of green, the dimerization domains of the DsbC monomers are shown in different shades of blue and the alpha domain of DsbD is shown in red. The actives sites of the DsbC monomers are shown in stick representation and the intermolecular disulphide bond between one active site Cys98 of DsbC and the active site Cys109 of DsbD is also shown (PDB:1ZJD). (e). Surface representation of DsbA with a small molecule fragment (PDB: 4WF4) bound shown in blue stick representation and a peptide bound (PDB: 4TKY) shown in white stick representation.

Figure 2. Peptidyl-proly cis/trans isomerases (a) Secondary structure of the dimeric macrophage infectivity potentiator from Legionella pneumophila (PDB: 2FD9). The dimerization domains are coloured in different shades of blue and the FKBP-like domains are coloured in different shades of salmon. (b) Molecular surface representation of monomeric MIP from Burkholderia pseudomallei (PDB: 2Y78) with the electrostatic charges mapped to 
the surface coloured from $-10 \mathrm{kT}$ (red) to $+10 \mathrm{kT}$ (blue). The substrate binding pocket is shown by the ellipse. (c) Surface representation of the FKBP-domain of MIP from Legionella pneumophila as a complex with rapamycin (PDB: 2VCD). Binding site residues and rapamycin are shown in green and white stick representation respectively. (d) Surface representation of Mip from Burkholderia pseudomallei as a complex with a pipecolic acid derivative (CJ40) (PDB: 4GGQ). The two conformations of CJ40 observed in the different molecules in the asymmetric unit are shown in ball and stick representation as white and yellows sticks. Key substrate binding residues are also detailed.

Figure 3. Peptidoglycan biosynthesis enzymes. (a) General reaction scheme of MurC-F family of amino acid ligases showing the enzyme intermediate formed. (b) Secondary structure representation of MurC (PDB: 1P3D). The 3 domains are coloured differently and labelled. The bound $\mathrm{Mn}^{2+}$ ions are shown in orange spheres and the bound UDP-N-acetylmuramoyl-Lalanine (UMA) and the non-hydrolysable ATP analogue, AMPPNP, are shown in ball and stick representation as white and yellow coloured bonds respectively. (c) Close up of the surface representation showing the binding sites for UMA in yellow sticks and AMPPNP in white stick representation. (d). Surface representation of MurD (PDB:2UUP) with a bound sulphonamide transition state analogue (17l) shown in ball and stick representation. Key residues making contacts with the inhibitor are detailed. Hydrogen bond contacts are shown in magenta dashed lines.

Figure 4. Lipopolysaccharide modification enzymes. (a) Chemical structure showing the modifications by EptA (addition of phosphoethanolamine) with red and green circles and by ArnT (addition of L-Ara4N) with the blue circle. (b). Ribbon diagram of the structure of ArnT 
bound to UndP (PDB: 5F15). (c) Active site region of ArnT with catalytic and substrate binding residues detailed. The bound UndP molecule is shown as a ball and stick representation in yellow bonds. (d). Ribbon diagram of the structure of EptA (PDB: 5FGN). (e). Active site region of EptA with a modelled Thre-280-PEA intermediate. The bound $\mathrm{Zn}^{2+}$ ion is shown as an orange sphere. Hydrogen bond contacts are shown in magenta coloured dashed lines. 
(a)

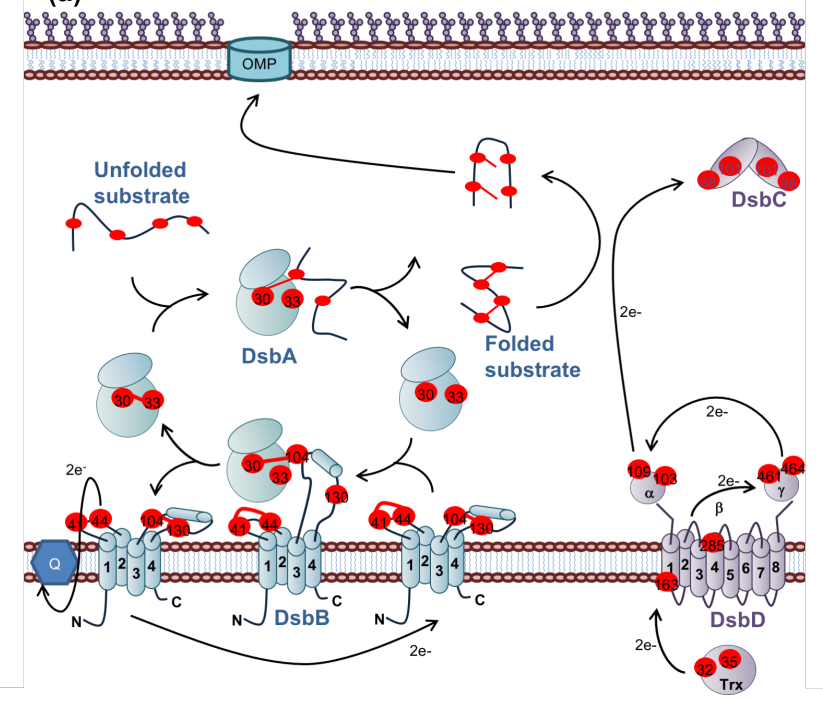

(b)

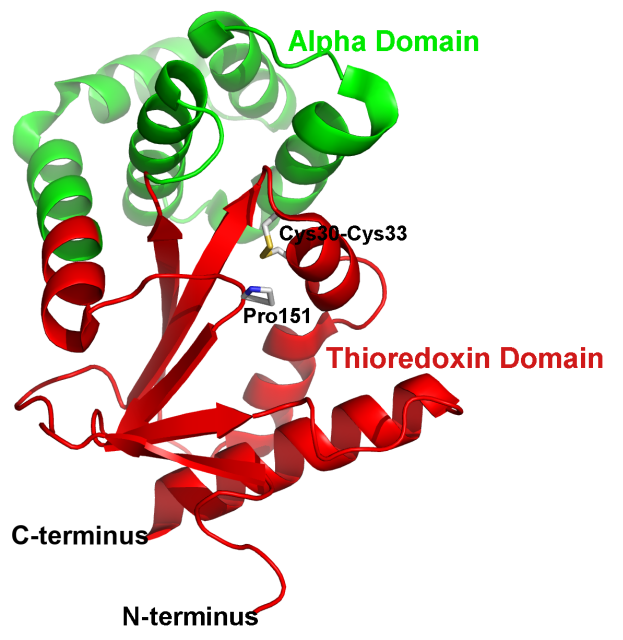

(c)

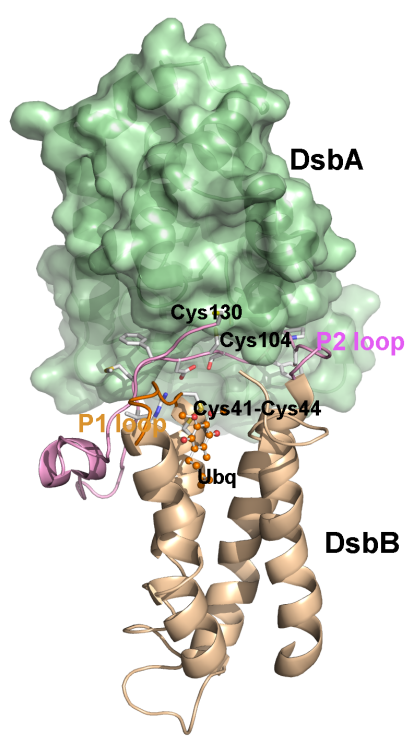

(d)
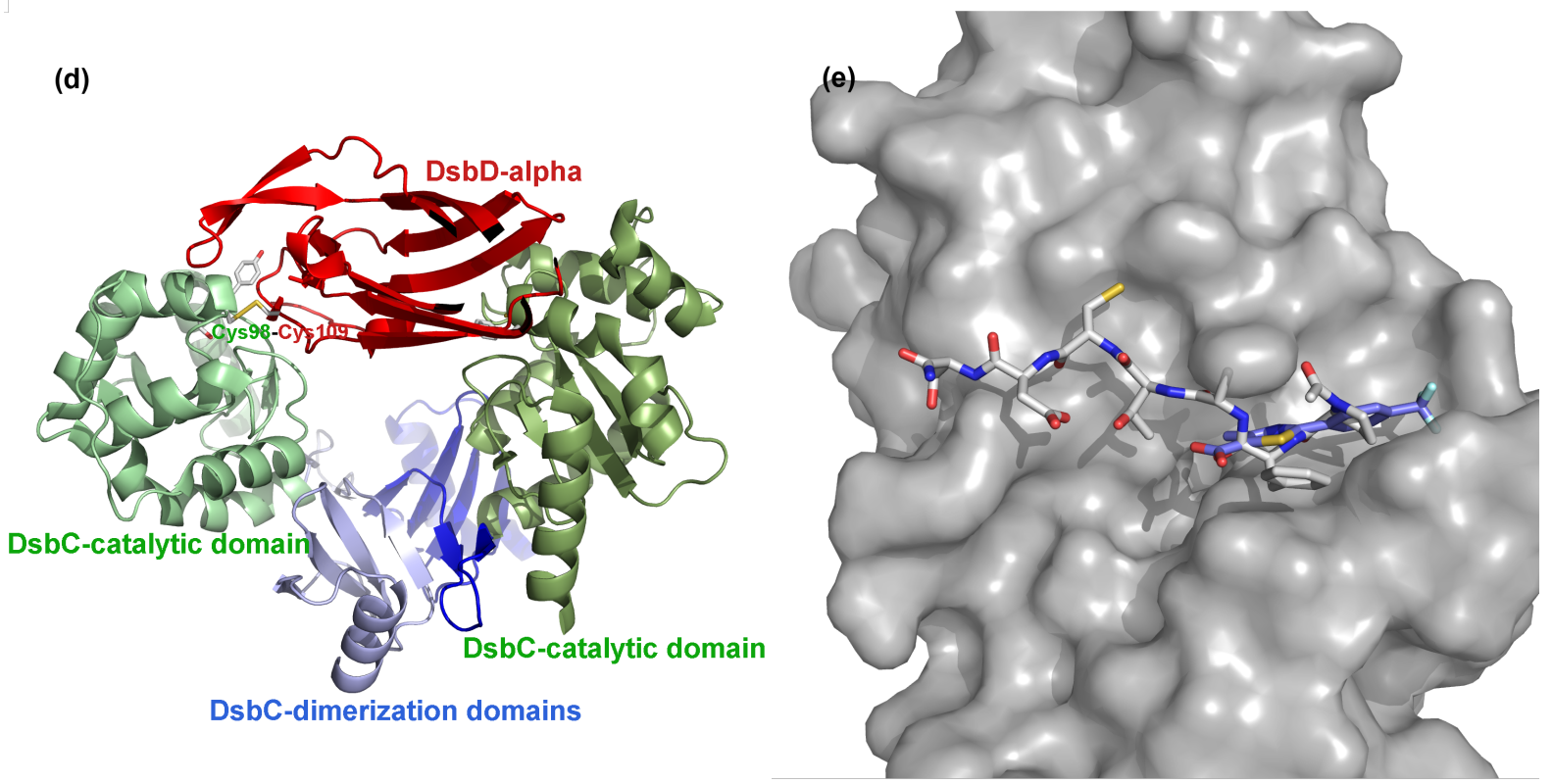

Figure 1 


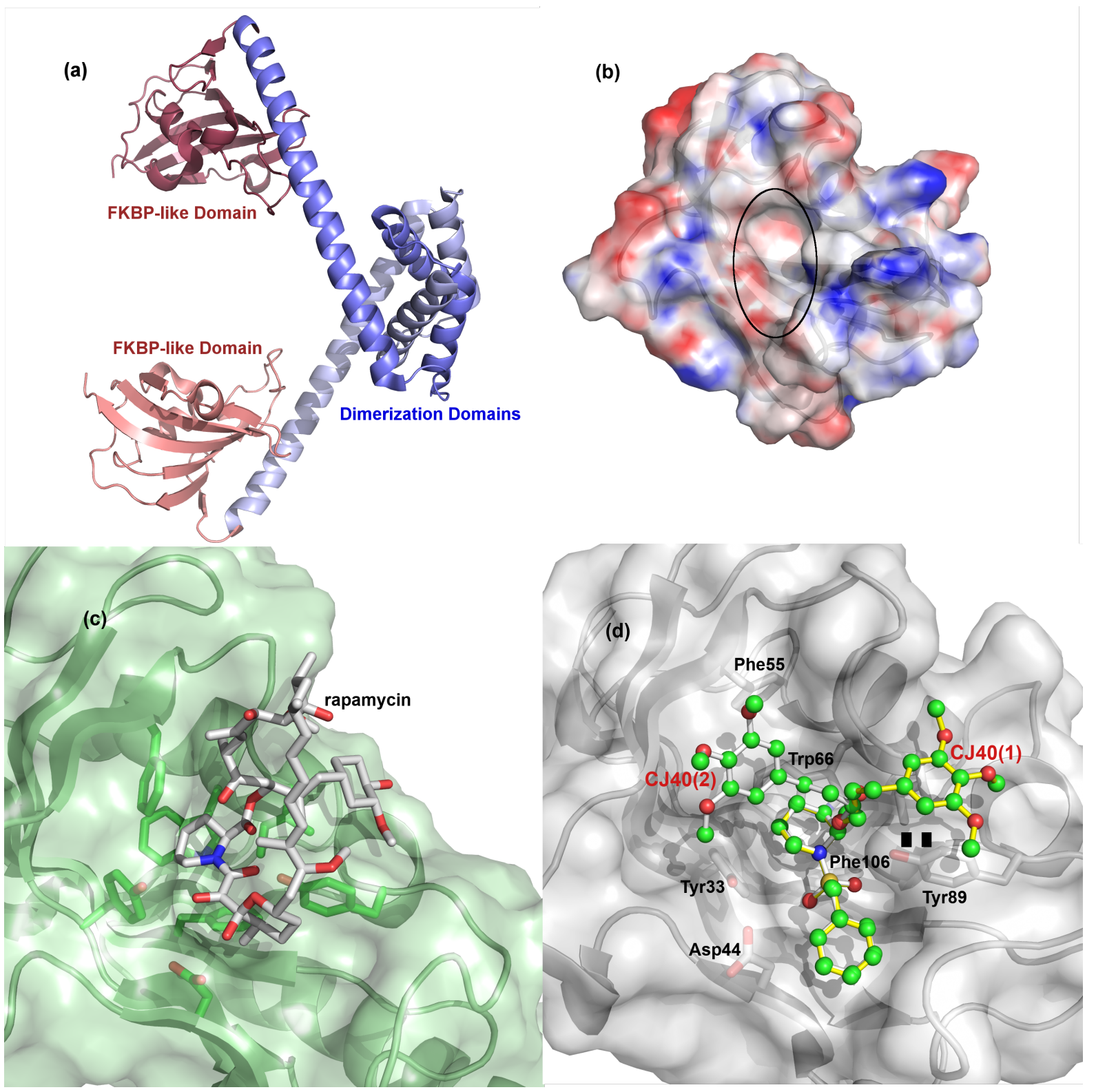

Figure 2 
(a)

$$
\begin{aligned}
& \mathrm{N}_{\text {Enzyme Intermediate }}^{\mathrm{ATP}} \\
& \begin{array}{lcc} 
& \mathbf{R} & \mathbf{R}^{\prime} \\
\text { MurC } & \text { L-alanine } & \text { UDP-N-acetylmuramic acid } \\
\text { MurD } & \text { D-glutamate } & \text { UDP- } N \text {-acetylmuramoyl-L-alanine } \\
\text { MurE } & \text { meso-diaminopimelate } & \text { UDP- } N \text {-acetylmuramoyl:tripeptide } \\
\text { MurF } & \text { D-alanine-D-alanine } & \text { UDP- } N \text {-aceylmuramoyl:pentapeptide }
\end{array}
\end{aligned}
$$

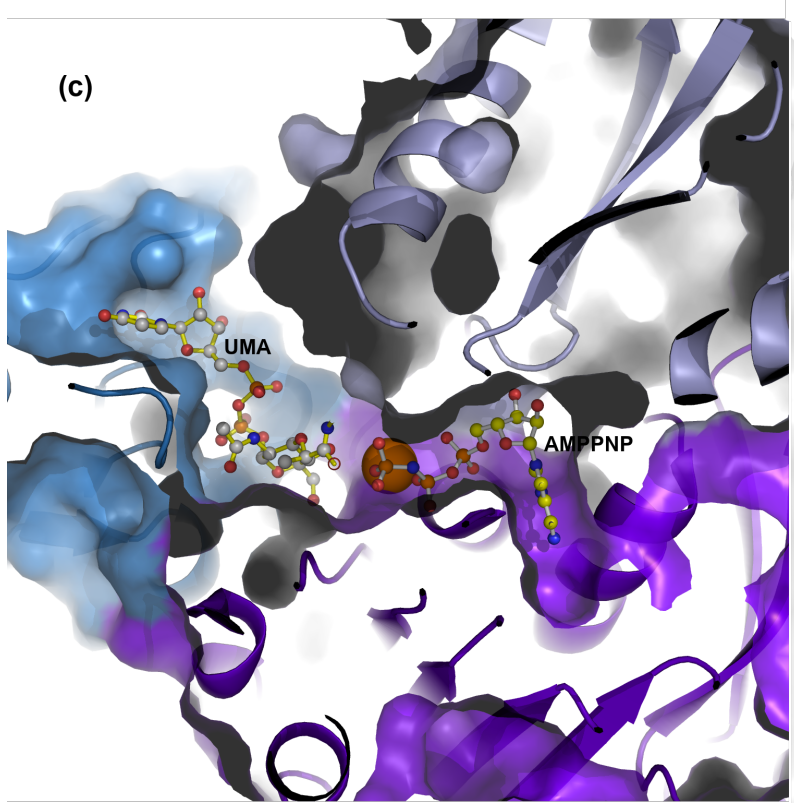

(b)

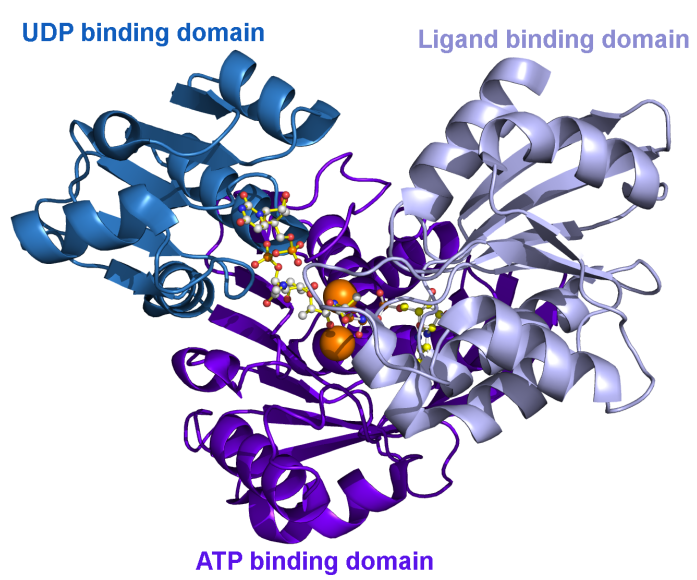

(d)

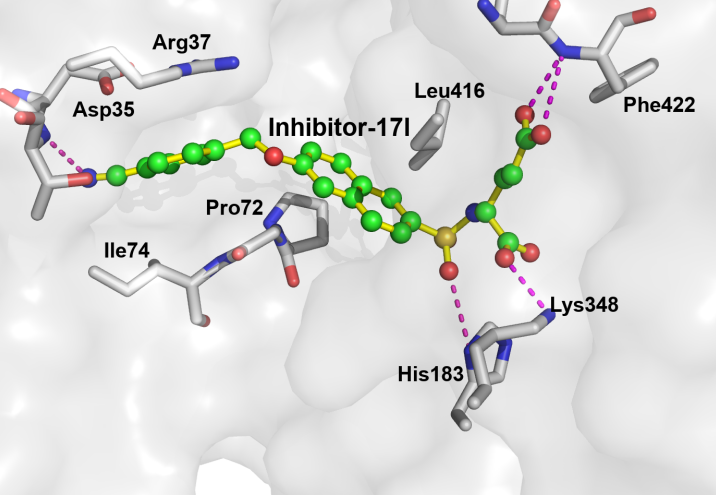

Figure 3 

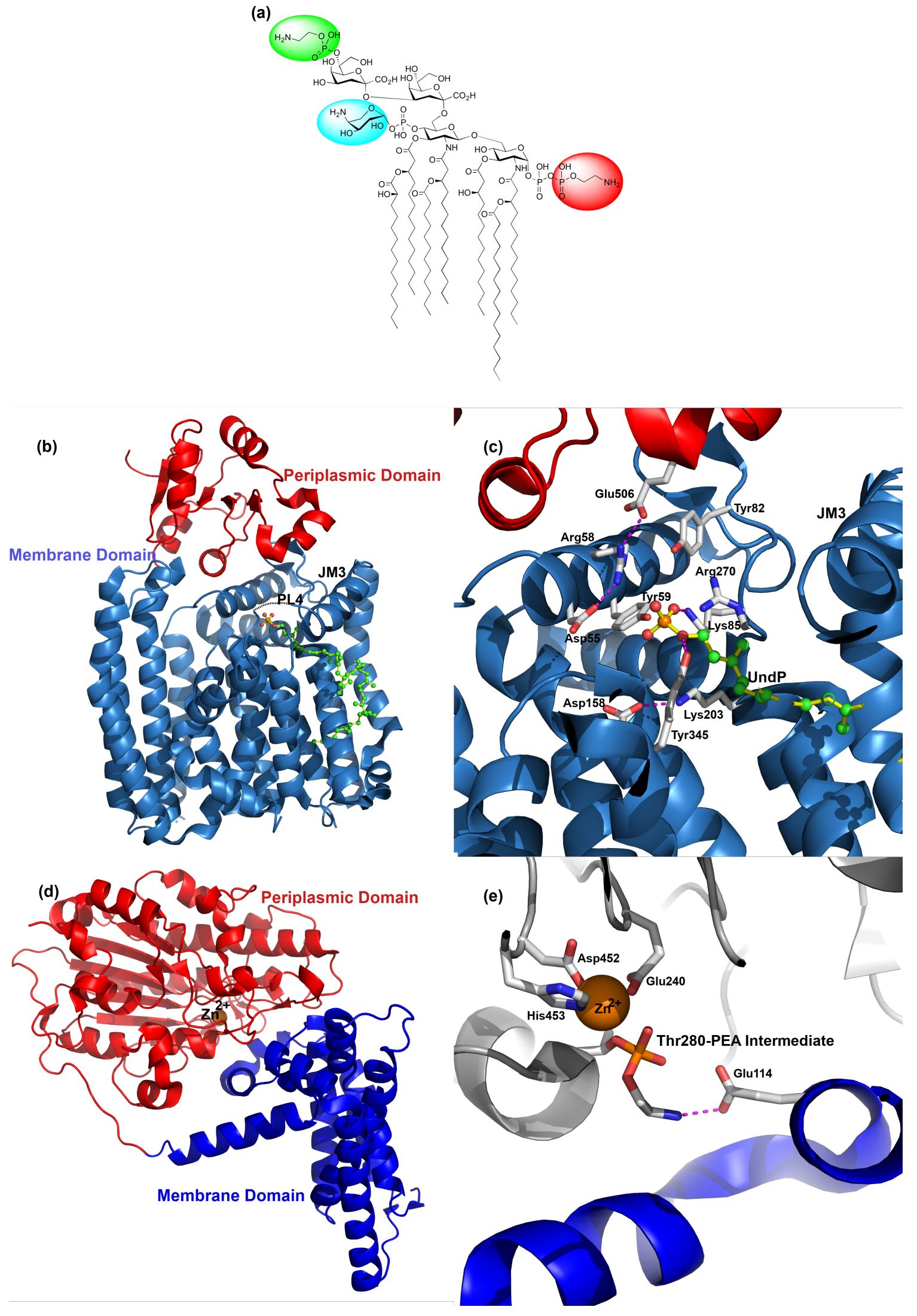

Figure 4 